\title{
Conference Paper \\ On the Role of Oxidation in Tribological Contacts under Environmental Conditions
}

\author{
Rolf Merz, Alexander Brodyanski, and Michael Kopnarski \\ Institut für Oberflächen- und Schichtanalytik GmbH (IFOS), Trippstadter Straße 120, 67663 Kaiserslautern, Germany
}

Correspondence should be addressed to Rolf Merz; merz@ifos.uni-kl.de

Received 31 July 2014; Accepted 26 November 2014

Academic Editor: Martin Dienwiebel

This Conference Paper is based on a presentation given by Rolf Merz at "European Symposium on Friction, Wear, and Wear Protection" held from 6 May 2014 to 8 May 2014 in Karlsruhe, Germany.

Copyright (C) 2015 Rolf Merz et al. This is an open access article distributed under the Creative Commons Attribution License, which permits unrestricted use, distribution, and reproduction in any medium, provided the original work is properly cited.

\begin{abstract}
Oxidation processes in tribological steel contacts are investigated, which are treated in a dry sliding, linear reciprocating model tribometer, by EDX (energy dispersive X-ray spectroscopy), AES (Auger electron spectroscopy), and HREFTEM (high resolution energy filtered transmission electron microscopy). Typical for steel contacts under environmental conditions is the feature that the counterparts are separated by oxide layers, which influence the tribological properties. And vice versa the tribological load will influence and change the oxide layers. The interaction of this dynamically coupled system was resolved by focussing the postexperimental surface analysis to long time stable balance states. As special challenge for the analyst of the tribological experiment under environmental conditions a postexperimental grown oxide layer covers the tribological induced changes and has to be distinguished from the tribological induced changes. Thick oxide layers, formed during the tribological load, were observed, which start to grow in form of islands and at the end separate the metallic bulk materials of the counterparts completely and avoid direct metal-metal contact. Thicknesses up to some microns strength, exceeding native oxide layers by magnitudes, were reached. Ploughing under fresh surface oxide and compacting and embedding of fresh oxidized debris particles were identified as main mechanisms responsible for the growing of these thick oxide layers.
\end{abstract}

\section{Introduction}

It is well known that the friction coefficient of metals is reduced due to oxide layer formation [1-6]. In addition, ceramics, which are in their chemical nature metal oxides, are widely used as protective layers in tribological contacts to reduce friction and increase endurance [7].

Typical native oxide layers formed, for example, on stainless steel surfaces under environmental conditions have only a thickness of a few Nanometers. In comparison technical samples even after high gloss polishing have still a surface roughness of approximately $0.1 \mu \mathrm{m}$. In direct contact surface structures like small asperity contacts jack the thin oxide layers through and direct intermetallic contacts are formed.

In contrast even in the case of mild wear of steel the growing of up to some microns thick oxide layers is observed, which separate the metallic counterparts [6]. Different mechanisms responsible for the forming are discussed in literature.

In Quinn's theory on the in situ oxidation of metals during sliding [8-12] locally higher contact temperatures push oxidation. If the oxide layer thickness exceeds a critical value it will break up and form debris particles. In consequence the wear rate depends on the oxidation rate. But the parabolic rate law for the oxidation process according to pure diffusion of oxygen through a growing oxide layer is not applicable in cases of mild wear [13].

In contrast, the forming of oxide layers due to compaction of oxide debris material is proposed as sufficient to explain the growing of thick oxide layers $[6,14]$. This was confirmed in the actual study. The growing of thick oxide islands is due to the embedding of oxidized debris particles. This was studied in detail by surface analysis. 


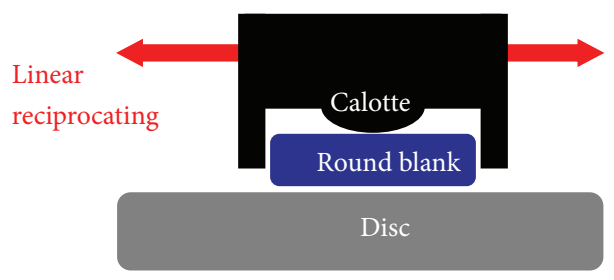

FiguRE 1: Experimental configuration of round blank versus disk tribometer.
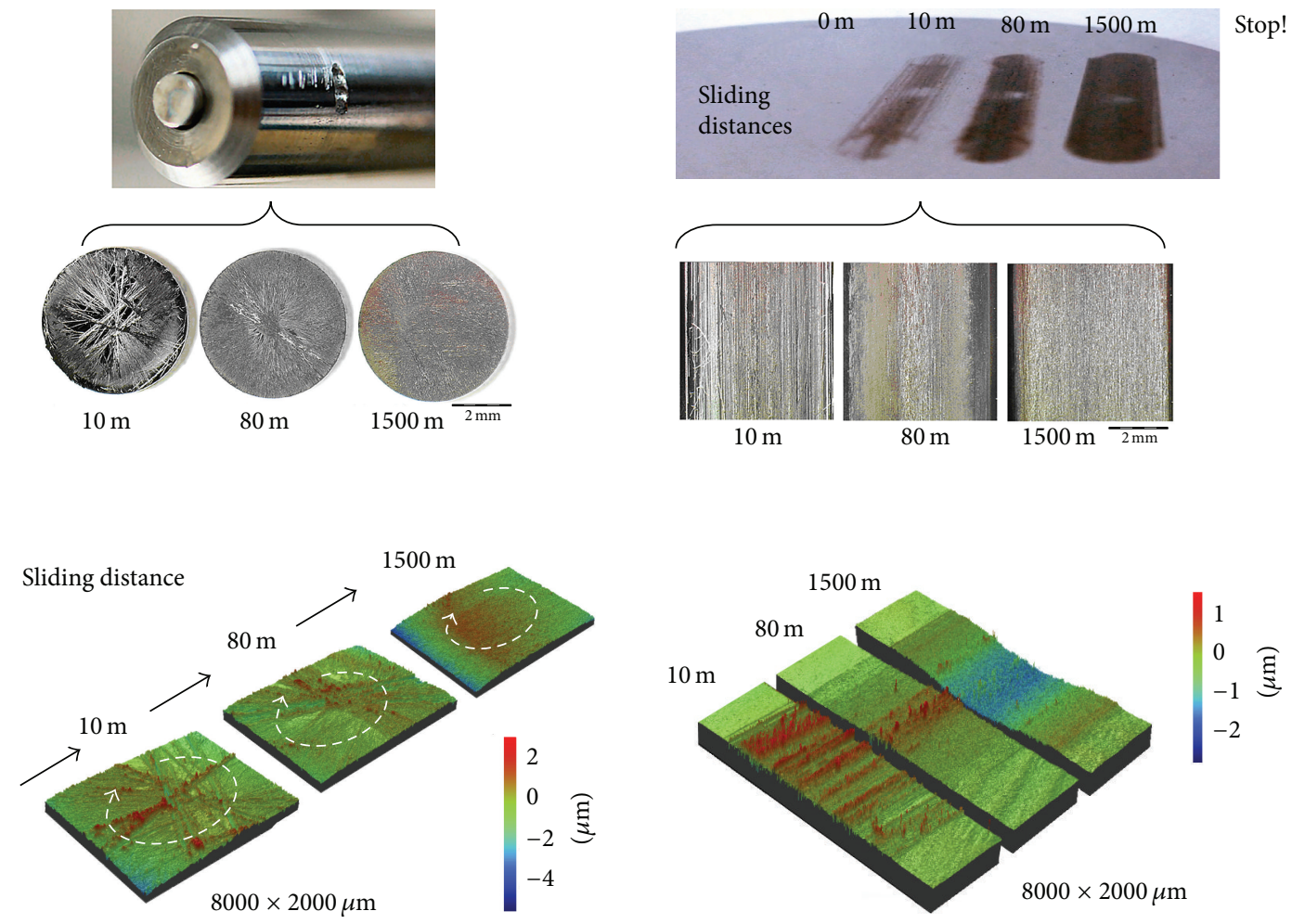

Wear marks on round blank

Linear wear track on disk

FIgURE 2: Appearance (dig. camera) and surface topography (white light interferometry) in 3D representation of round blank samples and counterparts after linear oscillating tribological load after the different sliding distances $(10 \mathrm{~m}, 80 \mathrm{~m}$, and $1500 \mathrm{~m})$.

\section{Experimental}

2.1. Tribometer. The tribometer tests were done with a pinon-disc model tribometer (CSM Instruments) in linear reciprocating configuration.

Mild wear of dry sliding steel was analyzed, with two parallel contact areas. The counterparts were $\varnothing 4 \mathrm{~mm}$ polished steel (100Cr6) round and a polished steel (100Cr6) plane disk. Figure 1 shows the configuration of the contact region. Runner is the small round blank underpinned by a calotte, which allows smoothly adaption of the tilt angles of the two contact areas. The normal load in the experiment was $2 \mathrm{~N}$; the sliding velocity was $1 \mathrm{~cm} / \mathrm{s}$.

The steel samples were polished and afterwards degreased in a three-level ultrasonic bath and purged with different cleaning agents (cyclohexane and acetone and isopropanol) in p.a. quality.
An additional scratch reference experiment without influence of oxygen was done in an ultrahigh vacuum apparatus. To do this a scratch unit was mounted directly inside the AES spectrometer (SMART 200 Scanning Auger Electron Microscope from Physical Electronics). A tungsten carbide scratch needle was fixed at the moveable sample transfer pole. So steel samples could be scratched with the needle under high vacuum conditions ( $p \sim 10^{-9}$ mbar) and immediately transferred to the analysis position without atmospheric venting. Repeating the analysis after controlled venting of the fresh scratch in the sample entry lock of the spectrometer enables isolation of effects of oxidation.

2.2. Analytical Techniques. Changes in surface near chemistry were analyzed by EDX (energy dispersive X-ray spectroscopy) and AES (Auger electron spectroscopy) in combination with sputter techniques. 

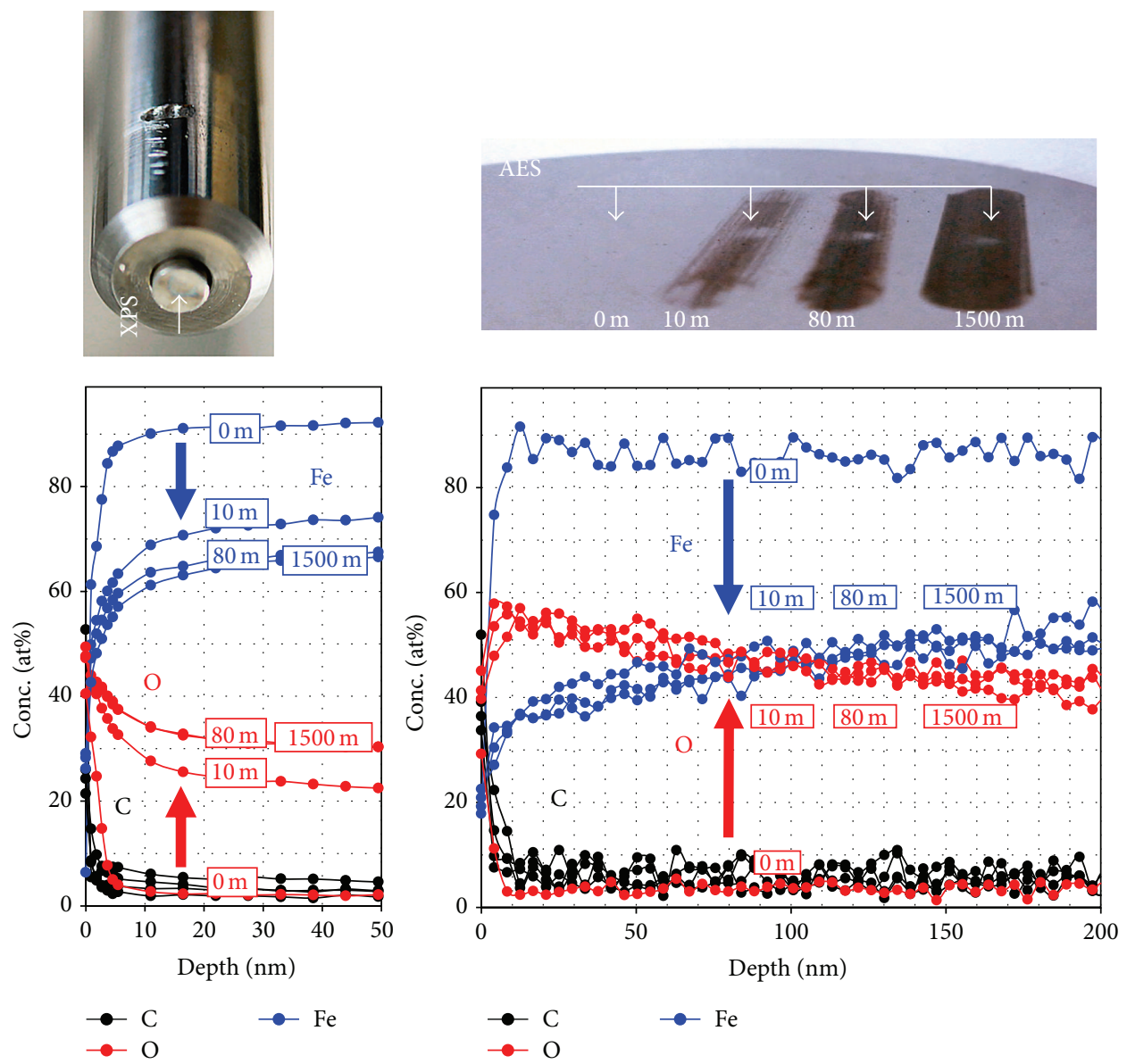

FiguRE 3: Surface chemistry in form of sputter depth profiles on round blank and disk after four different sliding distances $(0 \mathrm{~m}, 10 \mathrm{~m}, 80 \mathrm{~m}$, and $1500 \mathrm{~m})$.

The smaller information depth of AES (3-5 $\mathrm{nm}$ ) in contrast to $\operatorname{EDX}(>1 \mu \mathrm{m})$ [15] enables a better discrimination of even sub- $\mu$ debris particles, located inside a wear track, from the background beneath. Application of sputter techniques, that is, iterative removal of surface layers step by step by ion etching, in combination with surface analysis of the remaining surfaces after each step, gives the elemental depth distribution. Measurement of lateral elemental concentration maps after each sputter step extends the analysis to a $3 \mathrm{D}$ tomography containing the $3 \mathrm{D}$ elemental distribution of tribological contacts like previously demonstrated for hard metal sliding $[16,17]$.

Smaller particles were analyzed by high resolution energy filtered transmission electron microscopy (HREFTEM) with direct resolution of lattice planes. The gathered wear particles were further ultrasonically dispersed in isopropanol during a period of about 10 minutes. After that, they were fished on to the support copper TEM grid covered with formvar resin. The transmission electron microscopy (TEM) investigations were carried out using the Jeol 2010 TEM microscope (thermionic $\mathrm{LaB}_{6}$ cathode), an analytical configuration operating at $197 \mathrm{keV}$. The microscope is equipped by the slow-scan CCD camera (model MSC-794, Gatan), Gatan imaging filter (GIF-863 Tridiem), and an energy dispersive detector for registration and analysis of the X-ray induced by electrons (XEDS system, Oxford Instruments). An imaging was carried out in the energy filtered mode collecting only the elastic scattered electrons. The energy window of $10 \mathrm{eV}$ was symmetrically positioned around the zero-loss peak.

The chemical nature of the particles was identified according to their lattice spacing, determined by fast Fourier transform analysis and comparison to the database.

A cross section cut at the ground of a wear track was prepared by focused ion beam technique (FEI ALTURA 875 dualBeam FIB).

\section{Results and Discussion}

\subsection{Case of Mild Wear of Steel (Round Blank versus Disk)}

3.1.1. Topographical Changes. Figure 2 shows the appearance and $3 \mathrm{D}$ representation of the surface topography of the contact areas after three tribological experiments with different sliding distances $(10 \mathrm{~m}, 80 \mathrm{~m}$, and $1500 \mathrm{~m})$.

The round blank has the possibility to spin around, so the wear marks on the round blank are dominated during the running in phase by radial ridges and furrows. This degree of freedom will increase the wear in contrast to an angular fixed 

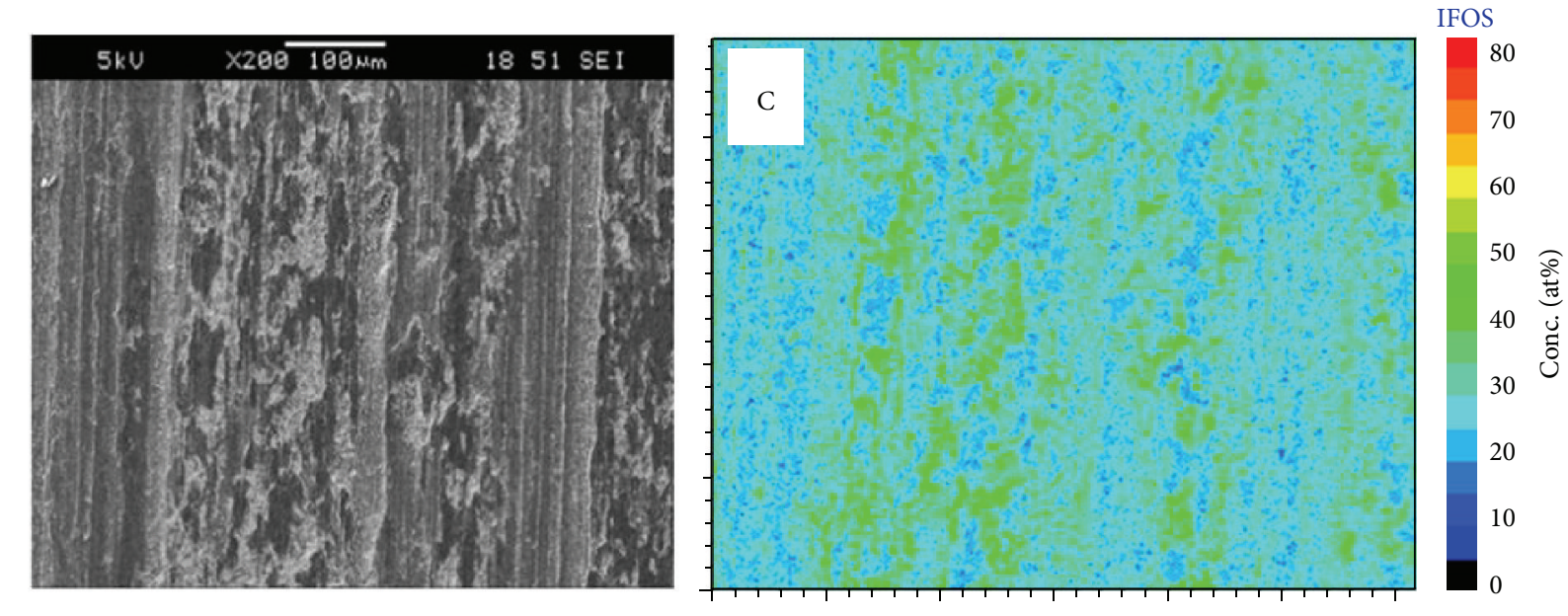

IFOS
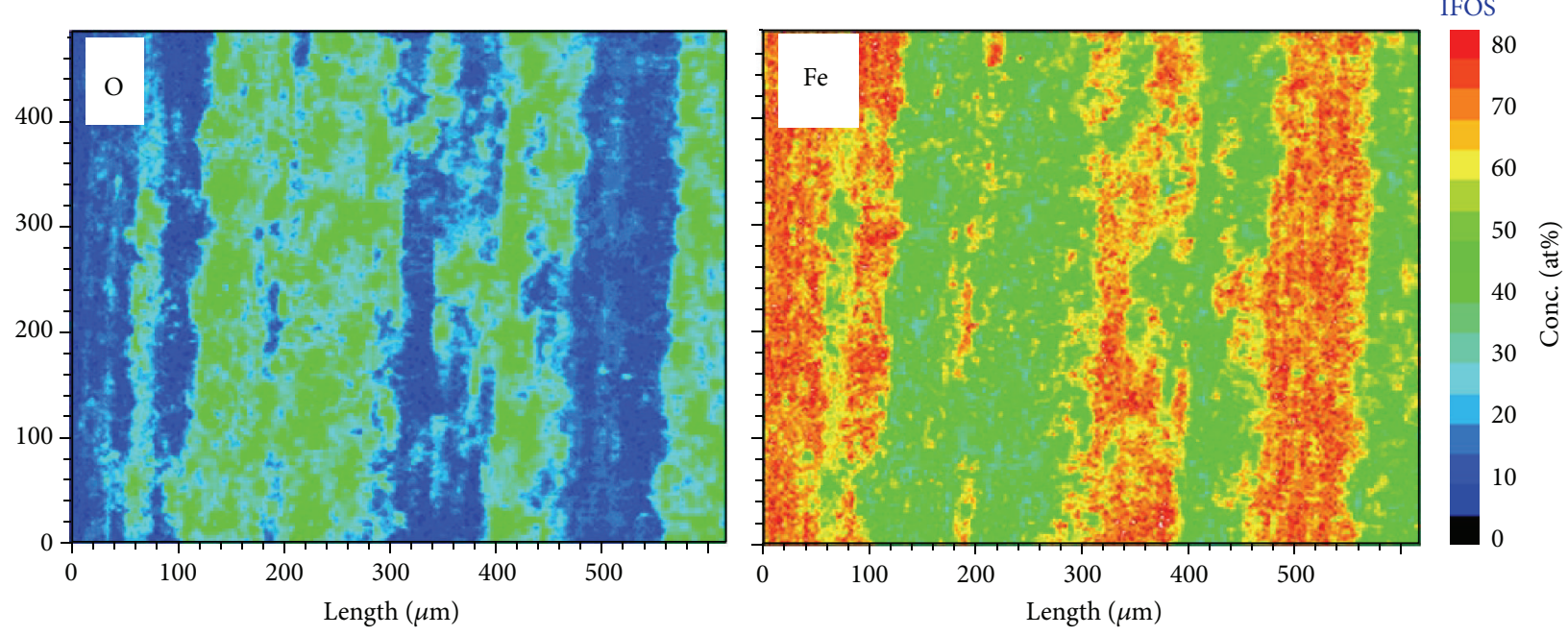

FIGURE 4: SEM and elemental concentration map of C, O, and Fe (at\%) of a wear track after a linear reciprocating tribological load (16MnCr5 versus $16 \mathrm{MnCr} 5)$ after $100 \mathrm{~m}$ sliding distance.

contact situation, because the thin ridges formed initially are sheared off after small turns of the round blank. After $1500 \mathrm{~m}$ sliding distance, the rough surface topography becomes smooth.

On the wear track of the counterpart during running in lots of debris material appears. It is piled up in form of linear ridges, oriented in sliding direction. After $1500 \mathrm{~m}$ sliding distance a broad valley with a wear depth of about $1.5 \mu \mathrm{m}$ and a length of $20 \mathrm{~mm}$ is formed and rarely loose debris material with easily observable size was detected. The smoothing out of debris material ridges with increasing sliding distance encourages already the assumption of embedding of debris material into the surfaces during overrunning by the slider.

The out-of-contact time for the disk is about $90 \%$ of the time for the round blank. The wear coefficient $(k)$ is calculated for the wear track as ratio of total wear volume $\left(W_{V}\right)$ through the product of normal load $\left(F_{N}\right)$ and relative sliding distance $(s)$ as $k=W_{V} /\left(F_{N} \cdot s\right)=4 \cdot 10^{-5} \mathrm{~mm}^{3} / \mathrm{Nm}$. Considering the total apparent contact areas $\left(A_{\mathrm{RB}}=12 \mathrm{~mm}^{2}\right)$ for the round blank and $\left(A_{D}=80 \mathrm{~mm}^{2}\right)$ for the disk, the average wear rate in terms of wear depth $(z)$ per sliding distance $(s)$, we find for the disk $\bar{w}=k \cdot F_{N} / A_{\mathrm{RB}}=1 \mathrm{~nm} / \mathrm{m}$ and $\bar{w}=k \cdot F_{N} / A_{D}=$ $7 \mathrm{~nm} / \mathrm{m}$ for the round blank.

3.1.2. Changes in Surface Chemistry. The surface chemistry of the top surface and the surface near region up to a depth of $50 \mathrm{~nm}$ (round blank), respectively, and $200 \mathrm{~nm}$ (disk) was investigated by electron spectroscopic sputter profiles (Figure 3) at an analysed spot covering the width of the wear track.

On the outer surface of both counterparts for all investigated sliding distances nearly the same oxygen, carbon, and iron concentration is detected. This is due to the exposition of the fresh surfaces to environmental oxygen, respectively, and adsorption of organic hydrocarbon films due to environmental influences.

In the depth region under the top surface significant differences appear with increasing sliding distance. The oxygen level over the investigated depth range grows significantly up to concentrations in the range of about 50 at $\%$. 

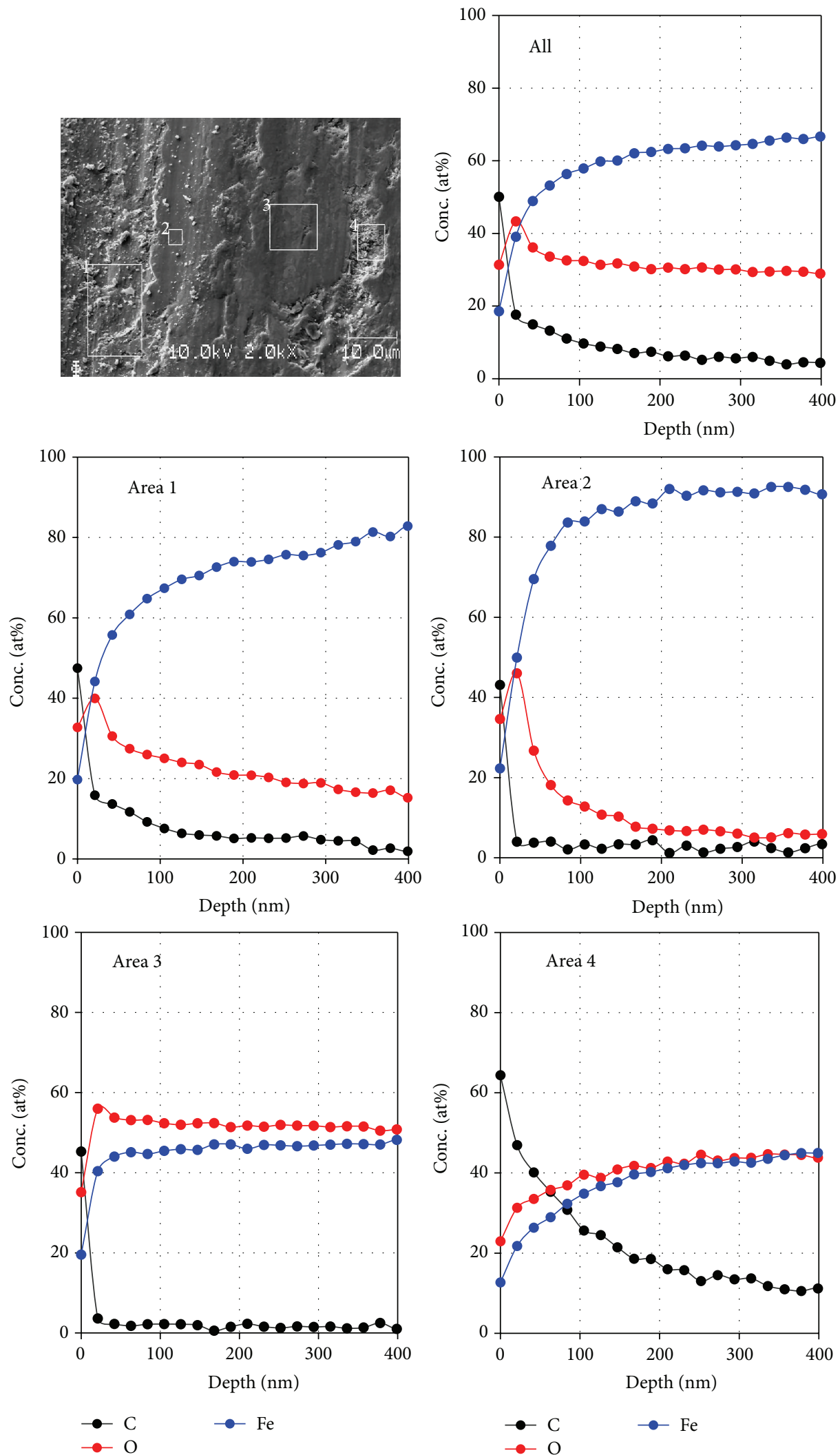

FIGURE 5: AES sputter depth profiles inside wear track, with high lateral resolution, measured over area regions 1-4, and over the whole SEM image area "All." 


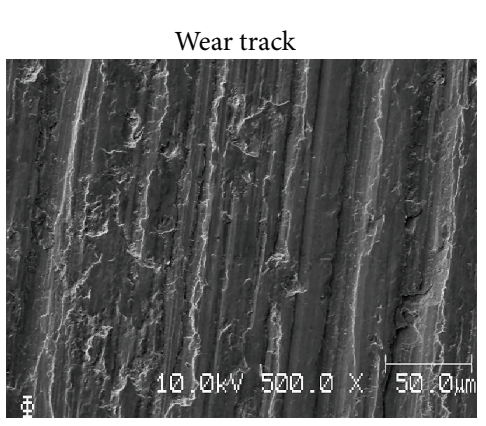

Wear track, after removal of debris particles by adhesive tape

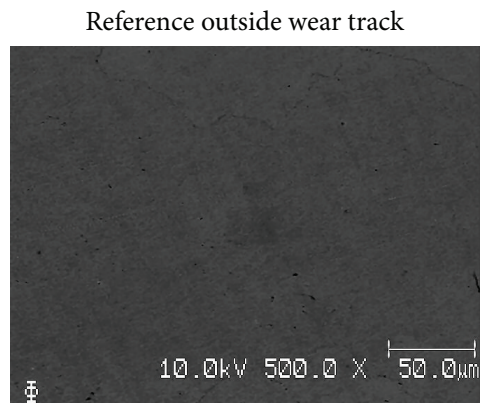

(a)
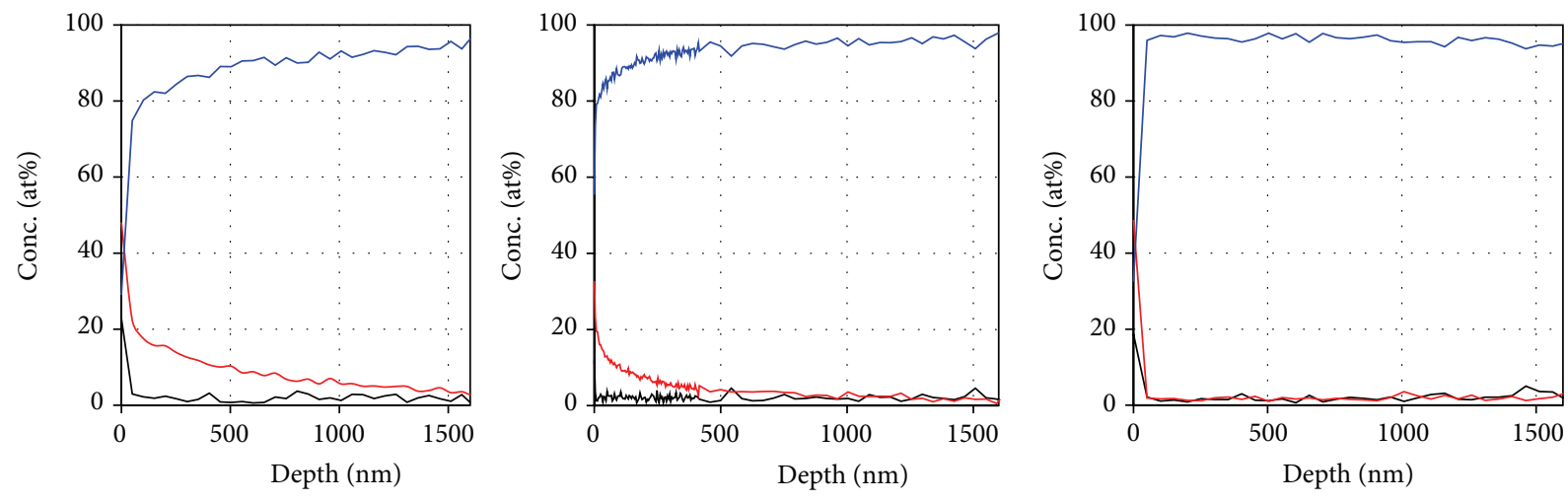

$-\mathrm{C}$

$-\mathrm{C}$

$-\mathrm{Fe}$

$-\mathrm{C}$

$-\mathrm{Fe}$

(b)

FIGURE 6: AES sputter profiles (b), measured over the area of the SEM pictures (a) before and after removal of debris particles by adhesive tape.
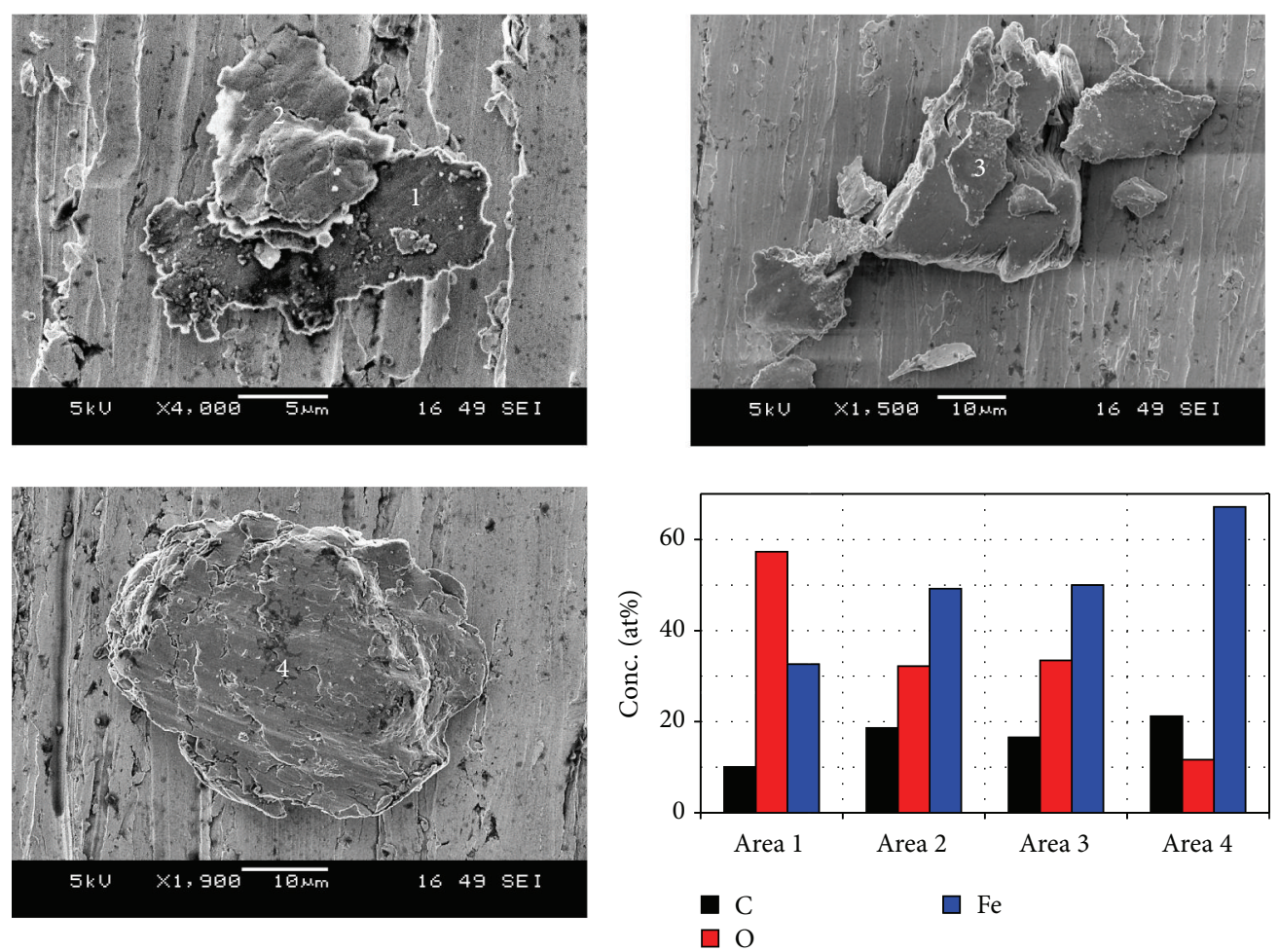

FIGURE 7: SE pictures of typical "bigger" debris particles and elemental concentrations (at\%). 

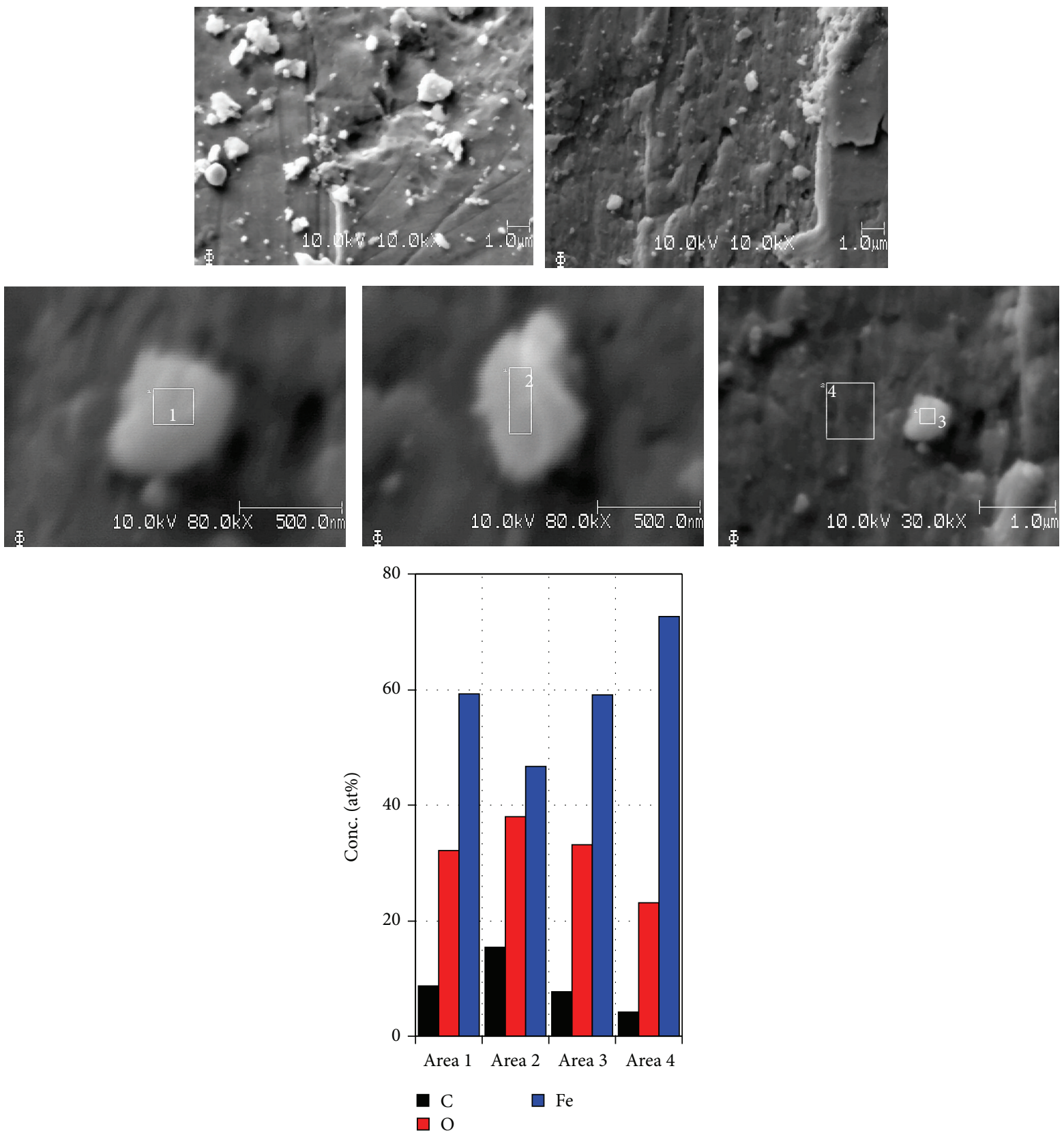

FIGURE 8: SEM pictures of sub- $\mu$ particles in the centre of the wear track after sliding, detected by AES spectroscopy and elemental concentrations in at $\%$.

The observed increase of oxygen concentration level is interpreted in accordance with Wilson et al. [6] as caused by a rising size and portion of thick oxidized islands (diameter $<\varnothing 500 \mu \mathrm{m}$ ) inside the analyzed area. Following Wilson et al. it is formed by oxidized, embedded, and compacted debris material.

After $100 \mathrm{~m}$ sliding distance the inhomogeneous oxygen and iron distribution, as shown in the elemental concentration maps determined by EDX (Figure 4) inside the wear track, reveals the oxide islands which grow more and more together with increasing sliding distance.

Sputter depth profiles up to a depth of $400 \mathrm{~nm}$ of four different area regions selected from the areas shown by the SEM images of the wear track after $100 \mathrm{~m}$ sliding distance were measured by Auger electron spectroscopy (Figure 5).

While in area 3 (slightly darker in the SE picture) a thick oxidized island appears as smooth area region where the debris material is already embedded and compacted, in area 2 (slightly brighter) only thin oxide layers were detected. Other regions (1 or 4) still are loaded with lots of even loose debris particles, which are partly or completely oxidized. AES sputter profiling with insufficient lateral resolution is not able to reveal these lateral differences (see Figure 5 "All").

To demonstrate the influence of debris particles on the detected oxygen level, AES sputter profiles are measured inside the wear track before and after removal of loose debris 


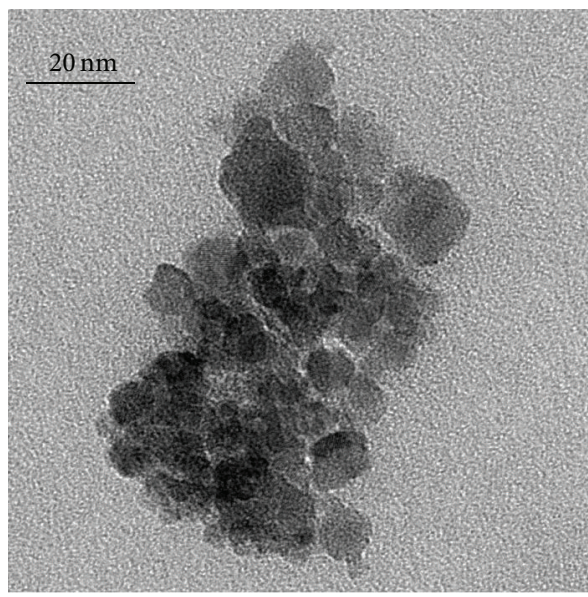

(a)

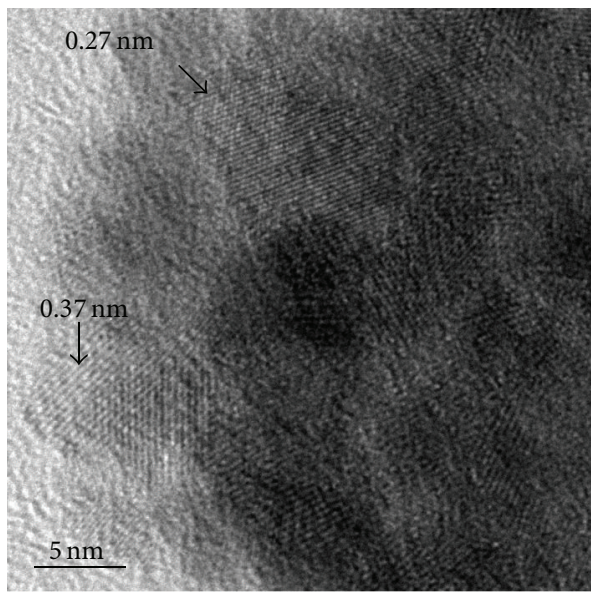

(c)

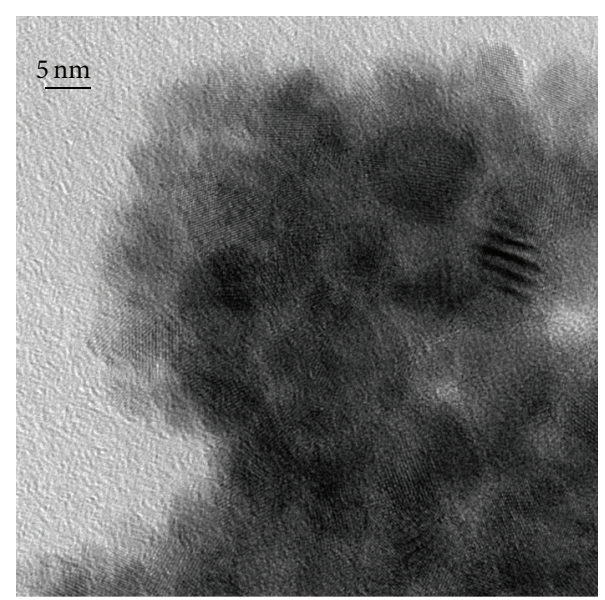

(b)

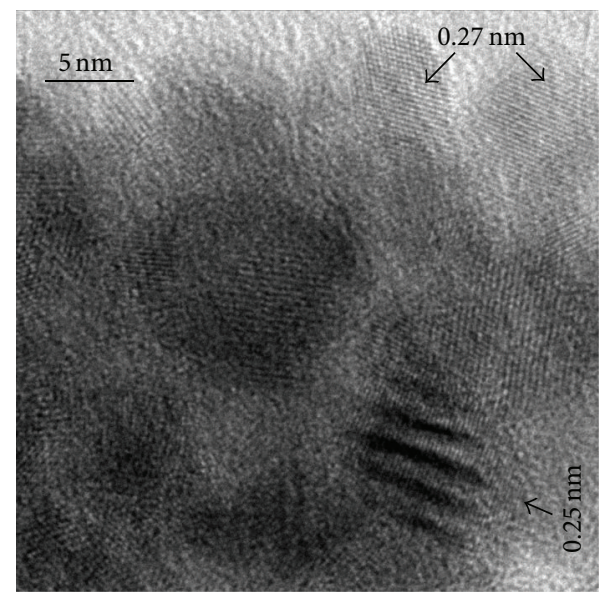

(d)

Figure 9: High resolution energy filtered TEM (HREFTEM) pictures with direct resolution of lattice planes of selected debris particles. According to database the lattice spacing found by fast Fourier transform analysis of this picture corresponds to the strongest lines of hematite $\left(\mathrm{Fe}_{2} \mathrm{O}_{3}\right)$.

particles by adhesive tape (Figure 6). A much steeper decrease in the oxygen depth profile is found after removal of the debris material. This supports the assumption that compared to the material of the wear track beneath the loose debris material is oxidized stronger.

3.1.3. Analysis of Debris Particles. After $100 \mathrm{~m}$ sliding distance, there is still a broad size spectrum of debris particles, which requires different methods of analysis. Some microns large particles are accessible to EDX (Figure 7), sub- $\mu$ particles are accessible to Auger analysis (Figure 8), and smaller ones demand high resolution energy filtered TEM analysis (Figure 9).

The EDX analysis (Figure 7) of some microns big debris particles shows particles with quite different degrees of oxidation. Partly and even completely oxidized particles are available for embedding. The Auger analysis (Figure 8) of smaller sub- $\mu$ particles demonstrates that the majority of these particles are oxidized stronger than the surface beneath, but still not completely oxidized.
At this particle size the discrimination of elemental contributions from particle and bulk material beneath becomes already difficult, so smaller particles were analyzed by HREFTEM.

Exemplary particles were purged out of the wear track by ethanol and dried on a TEM grid. It was established that the wear particles collected on the TEM grid are very small and possess a dimension of about (or smaller) $100 \mathrm{~nm}$. Under light microscope (magnification up to 2000 -fold) no particles are visible on the TEM grid.

It can be seen (Figure 9) that the wear particle is actually an agglomeration consisting of many nanoparticles, in which size varies from about 8 to $18 \mathrm{~nm}$. The lines seen within the nanoparticles (line spacing of about $0.38 \mathrm{~nm}$ and more) in the left image constitute so-called moiré patterns caused by overlapping two or more nanoparticles. The good visibility of the nanoparticles even in the agglomeration centre points to a thickness less than $50 \mathrm{~nm}$ based on our experience with the TEM investigations of iron specimens.

In parallel with the moiré patterns in the middle region of the agglomeration, the fast Fourier transform analysis of the 


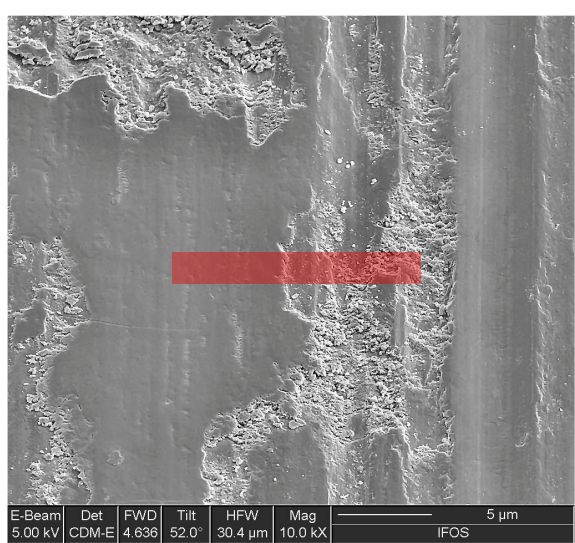

(a) SEM picture inside the centre of the wear track (position of cross section marked in red)

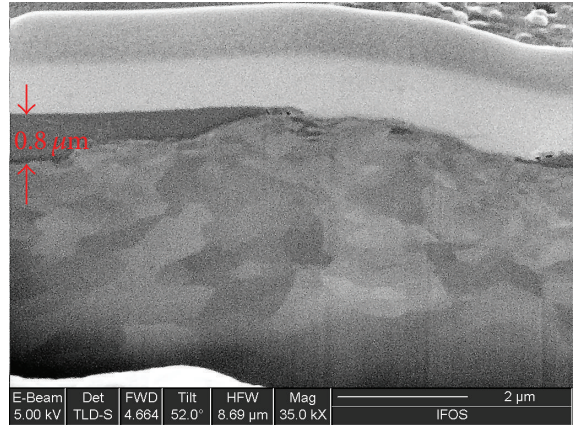

(c) SEM picture of the cross section

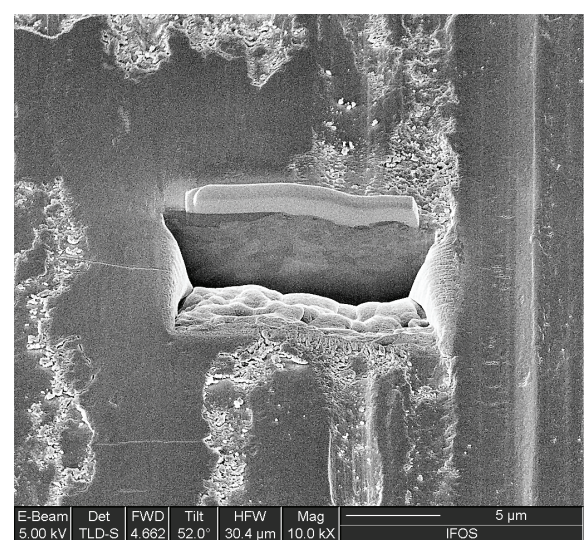

(b) SEM picture of the cross section, prepared by focused ion beam

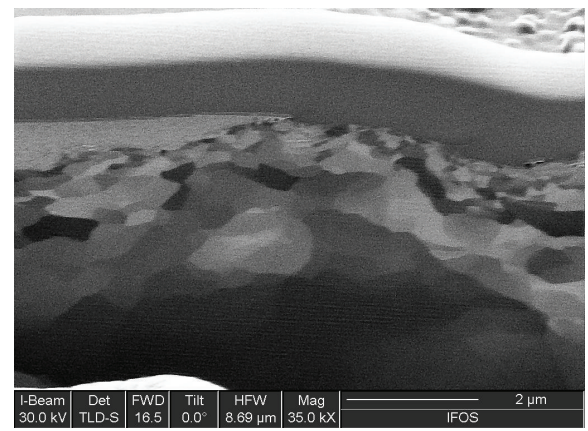

(d) SEM picture taken by ion induced secondary electrons

FIGURE 10: Illustration of FIB cut procedure.

high resolution images (Figure 9(b)) also reveals the genuine lattice spacing in the one-nanoparticle regions. These regions are mainly located on the periphery of the agglomeration but also often present in the centre part of the agglomeration (see the nanoparticles marked by arrows). Three values of the lattice spacing are established for these particles: $0.37 \mathrm{~nm}$, $0.27 \mathrm{~nm}$, and $0.25 \mathrm{~nm}$. The lattice spacing observed by us agrees well with the first biggest lattice spacing of the iron oxides in the hematite phase. This lattice spacing causes first three strongest diffraction reflexes of hematite and, therefore, is the best recognizable line spacing in the HRTEM images. The nanoparticles with such line spacing are observed practically everywhere within the agglomeration. It means that the hematite nanoparticles constitute a main part of the investigated wear particles.

In Figure 10 a cross section cut at the ground of the wear track is shown, which was prepared by focused ion beam technique (FEI ALTURA 875 dualBeam FIB). The cut was made perpendicular to the sliding direction at a surface region appearing similar to area 3 of Figure 5, which was interpreted as smoothed area region where debris material is already embedded and compacted. In the cross section pictures of Figure 10 a thickness of this layer of 0.8 microns was found. In contrast to its surrounding no grain structure was observed in the ion induced SE pictures of this layer.
All these observations support the hypothesis that during sliding oxidized debris material in form of particles is compacted and embedded into the surface during sliding. Available for embedding are debris particles with different degrees of oxidation and different sizes from some microns down to Nanometer diameter. Particularly the smallest particles, which are formed during the reciprocating tribological load, by grinding of bigger particles, are completely oxidized by the environmental oxygen attack. The surface magnification due to grinding is sufficient to explain the amount of oxygen present in the wear track.

3.1.4. Influence of Environmental Oxygen. It can be easily demonstrated that the oxygen in the ambient air oxidizes the fresh created iron surface. For this purpose we scratched the steel surface inside the ultrahigh vacuum AES chamber and imaged the corresponding oxygen distribution before and after venting for 10 minutes at atmosphere (Figure 11). After venting the chemical traces of the scratch fade out in the chemical concentration maps with the time at atmosphere, because a thin film of fresh surface oxide covers the scratch. It is to be mentioned that the film of adsorbed hydrocarbons due to environmental influences grows slightly slower than the oxide layer. 

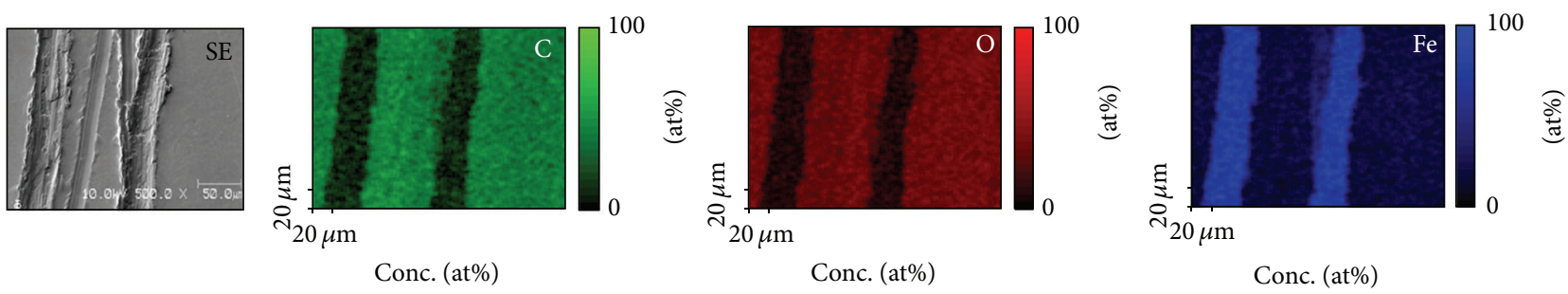

Fresh scratch, scratched and analyzed at $p \sim 10^{-9} \mathrm{mbar}$

(a)
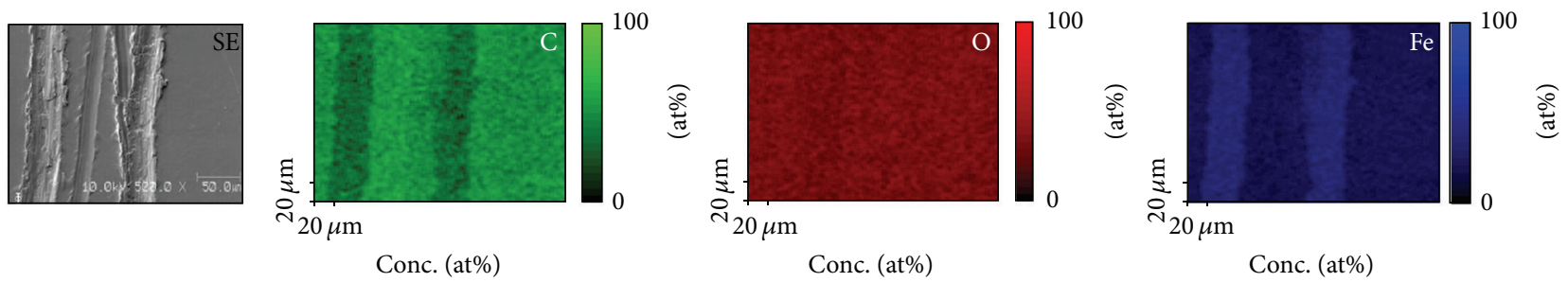

Same scratch, after 10 min venting at atmosphere $p \sim 10^{-3} \mathrm{mbar}$

(b)
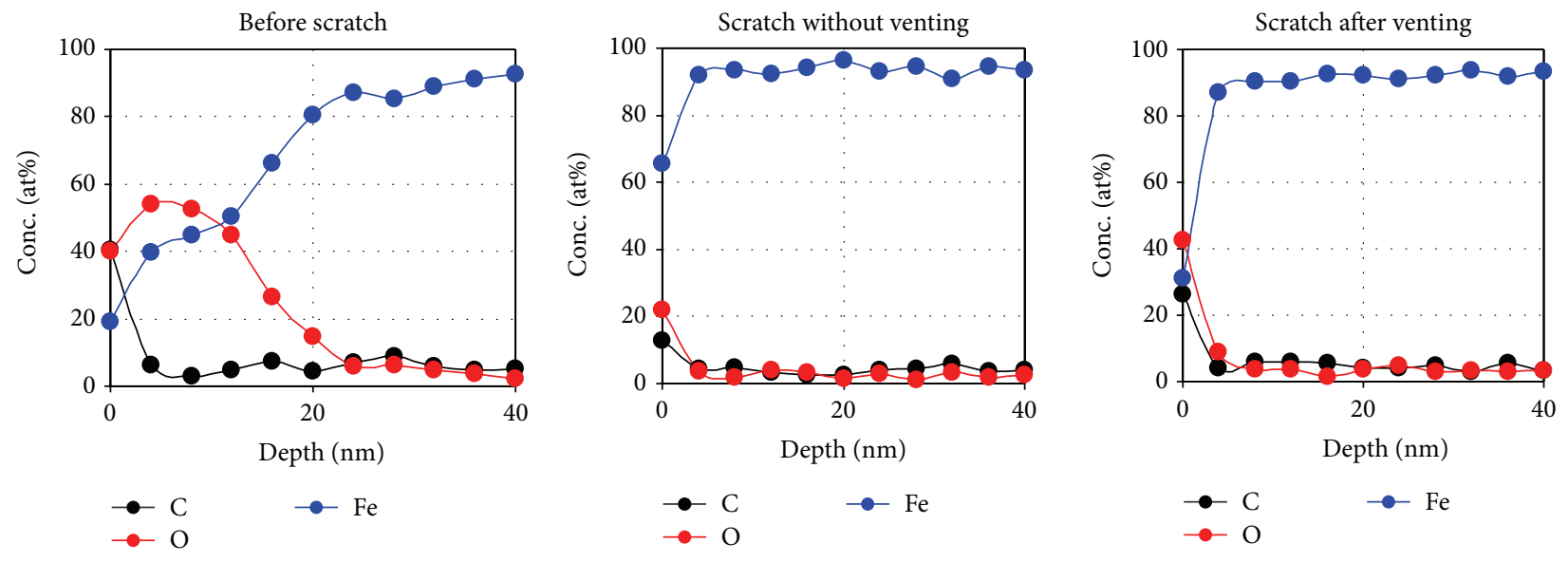

(c)

FIGURE 11: First and second line: SEM images and C, O, and Fe elemental concentration maps of a steel sample, measured by AES immediately after scratch under high vacuum conditions and after short venting at atmosphere (10 min). Last line: sputter depth profiles before scratch, after scratch under UHV, and after 10 min venting at environmental pressure.

The sputter depth profiles shown in Figure 11(c) clearly prove that the oxide layer thickness due to environmental oxidation is in the Nanometer range only, that is, within the information depth of AES.

The thick oxide islands, observed after the tribological reciprocating experiments, are performed in time scales comparable to the venting experiment. So the question concerning the source of oxide formation is arising.

Enhanced oxidation could be due to the high flash temperatures during the asperity contact. Even under atmospheric conditions after scratching of a clean metal surface with an only Nanometer thin oxide layer on top we could not find any indication of thicker oxide layers, which would support the assumption of enhanced oxidation during the asperity contact.
We conclude that the triboinduced enormous increase of the surface area is responsible. This effect is caused by the grinding of debris particles down to nanoparticle size during the reciprocating load. The majority of Nanometer sized particles identified as pure hematite by HREFTEM are embedded and compacted inside the wear track and constitute the observed oxide islands.

\section{Conclusion}

Oxygen plays a crucial role in tribological load of steel counterparts under environmental conditions.

During tribological stressing thick oxide layers grow up to a thickness of some microns. These layers are not closed, but in form of islands. Analysis with high lateral resolution 
capturing different area regions inside the wear track shows regions with thin native oxide films and others with thick oxidic layers and areas with accumulations of loose debris particles at the surface.

The mechanisms responsible for the forming of such thick oxidic layers are the ploughing under fresh surface oxide as well as the compacting and embedding of fresh oxidized debris particles. Tribological induced enhancement of oxidation by temperature effects seems to play a minor role under our conditions, that is, in the case of mild wear of steel.

Debris particles with sizes from some microns down to some Nanometers were detected. Quite different degrees of oxidation of the particles were found. The main fraction of particles, available for embedding in the tribological contact, was identified as clusters of completely oxidized (hematite) nanoparticles. Their complete oxidation is a consequence of grinding of debris material down in the Nanometer size and the correspondence increase of the specific surface.

\section{Conflict of Interests}

The authors declare that there is no conflict of interests regarding the publication of this paper.

\section{Acknowledgments}

The authors would like to thank the Deutsche Forschungsgemeinschaft. The research at IFOS is conducted within the Collaborative Research Centre (CRC 926): "Microscale Morphology of Component Surfaces (MICOS)" where the Institute for Surface and Thin Film Analysis (IFOS) is working on gaining a comprehensive knowledge of the interactions between chemical, structural, and topographical surface properties and friction and wear.

\section{References}

[1] E. Rabinowicz, "Lubrication of metal surfaces by oxide films," ASLE Transactions, vol. 10, no. 4, pp. 400-407, 1967.

[2] H. Kong, E.-S. Yoon, and O. K. Kwon, "Self-formation of protective oxide films at dry sliding mild steel surfaces under a medium vacuum," Wear, vol. 181-183, no. 1, pp. 325-333, 1995.

[3] H. So, "The mechanism of oxidational wear," Wear, vol. 184, no. 2, pp. 161-167, 1995.

[4] K. Kato, "Wear in relation to friction-a review," Wear, vol. 241, no. 2, pp. 151-157, 2000.

[5] S.-M. Du, Y.-Z. Zhang, Y. Chen, and W.-M. Liu, "The effect of oxygen on the tribological behavior of CrNiMo steel," Industrial Lubrication and Tribology, vol. 64, no. 2, pp. 71-76, 2012.

[6] J. E. Wilson, F. H. Stott, and G. C. Wood, "The development of wear protective oxides and their influence on Sliding friction," Proceedings of the Royal Society of London A, vol. 369, pp. 557$574,1980$.

[7] H. Kitsunai, K. Hokkirigawa, N. Tsumaki, and K. Kato, “Transitions of microscopic wear mechanism for $\mathrm{Cr}_{2} \mathrm{O}_{3}$ ceramic coatings during repeated sliding observed in a scanning électron microscope tribosystem," Wear, vol. 151, no. 2, pp. 279-289, 1991.

[8] T. F. J. Quinn, "Role of oxidation in the mild wear of steel," British Journal of Applied Physics, vol. 13, no. 1, pp. 33-37, 1962.
[9] T. F. J. Quinn, J. L. Sullivan, and D. M. Rawson, "New developments in the oxidational theory of the mild wear of steels," in Proceedings of the International Conference on Wear of Materials, K. C. Ludema, W. A. Glaeser, and S. K. Rhee, Eds., pp. 1-11, Publications of the American Society of Mechanical Engineering, Daerborn, Mich, USA, April 1979.

[10] T. F. J. Quinn, “Oxidational wear modelling: part I,” Wear, vol. 153, no. 1, pp. 179-200, 1992.

[11] T. F. J. Quinn, “Oxidational wear modelling: part II. The general theory of oxidational wear," Wear, vol. 175, no. 1-2, pp. 199-208, 1994.

[12] T. F. J. Quinn, "Oxidational wear modelling part III. The effects of speed and elevated temperatures," Wear, vol. 216, no. 2, pp. 262-275, 1998.

[13] H.-S. Hong, "The role of atmospheres and lubricants in the oxidational wear of metals," Tribology International, vol. 35, no. 11, pp. 725-729, 2002.

[14] A. Iwabuchi, "The role of oxide particles in the fretting wear of mild steel," Wear, vol. 151, no. 2, pp. 301-311, 1991.

[15] D. Briggs and J. Grant, Surface Analysis by Auger and X-Ray Photoelectron Spectroscopy, IM Publications, Chichester, UK; Surface Spectra, Manchester, UK, 2003.

[16] P. Stoyanov, P. A. Romero, R. Merz et al., "Nanoscale sliding friction phenomena at the interface of diamond-like carbon and tungsten," Acta Materialia, vol. 67, pp. 395-408, 2014.

[17] P. Stoyanov, P. Stemmer, T. T. Järvi et al., "Friction and wear mechanisms of tungsten-carbon systems: a comparison of dry and lubricated conditions," ACS Applied Materials \& Interfaces, vol. 5, no. 13, pp. 6123-6135, 2013. 

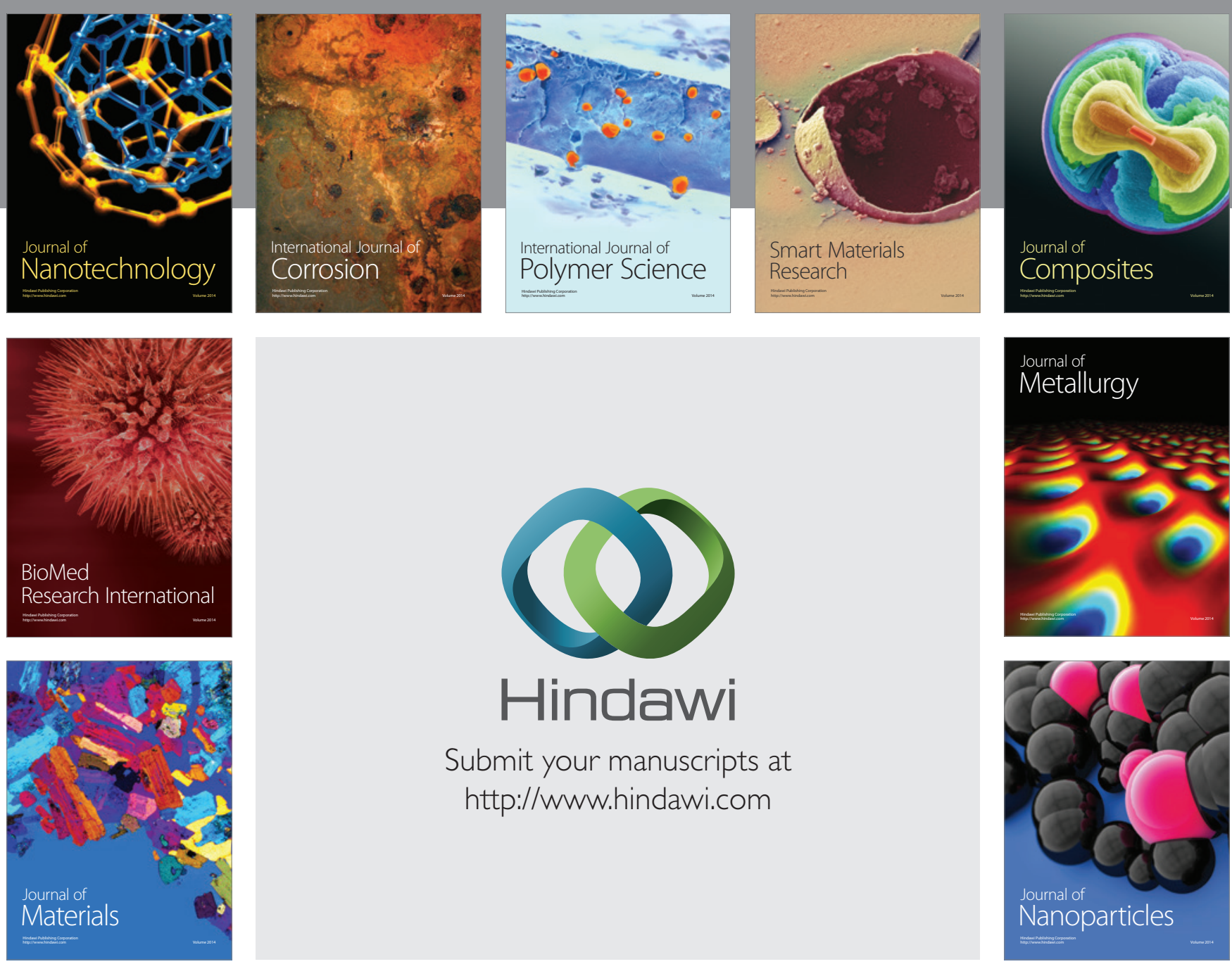

Submit your manuscripts at http://www.hindawi.com
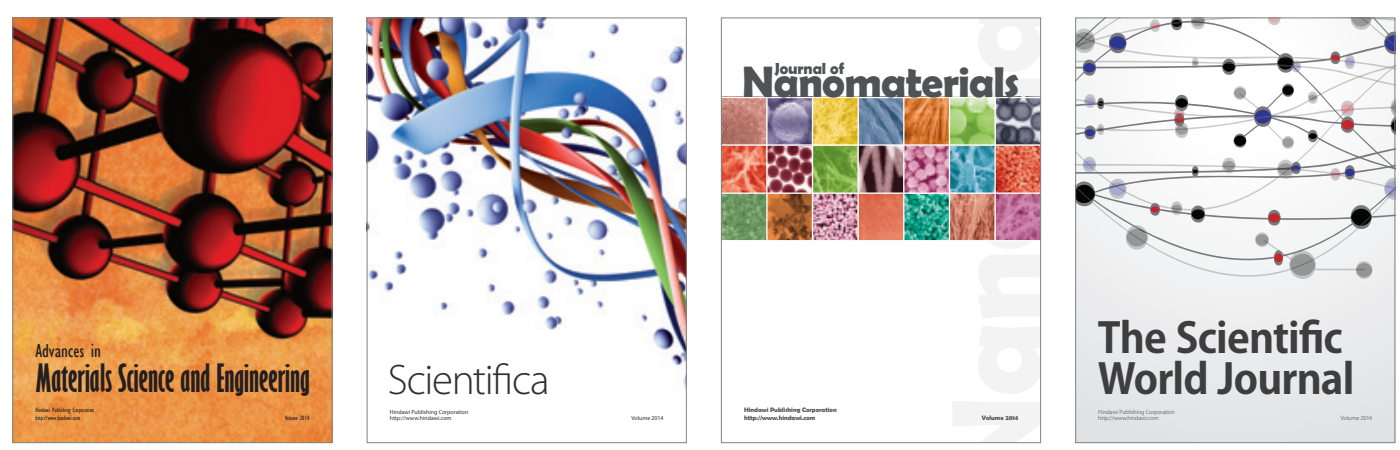

\section{The Scientific World Journal}
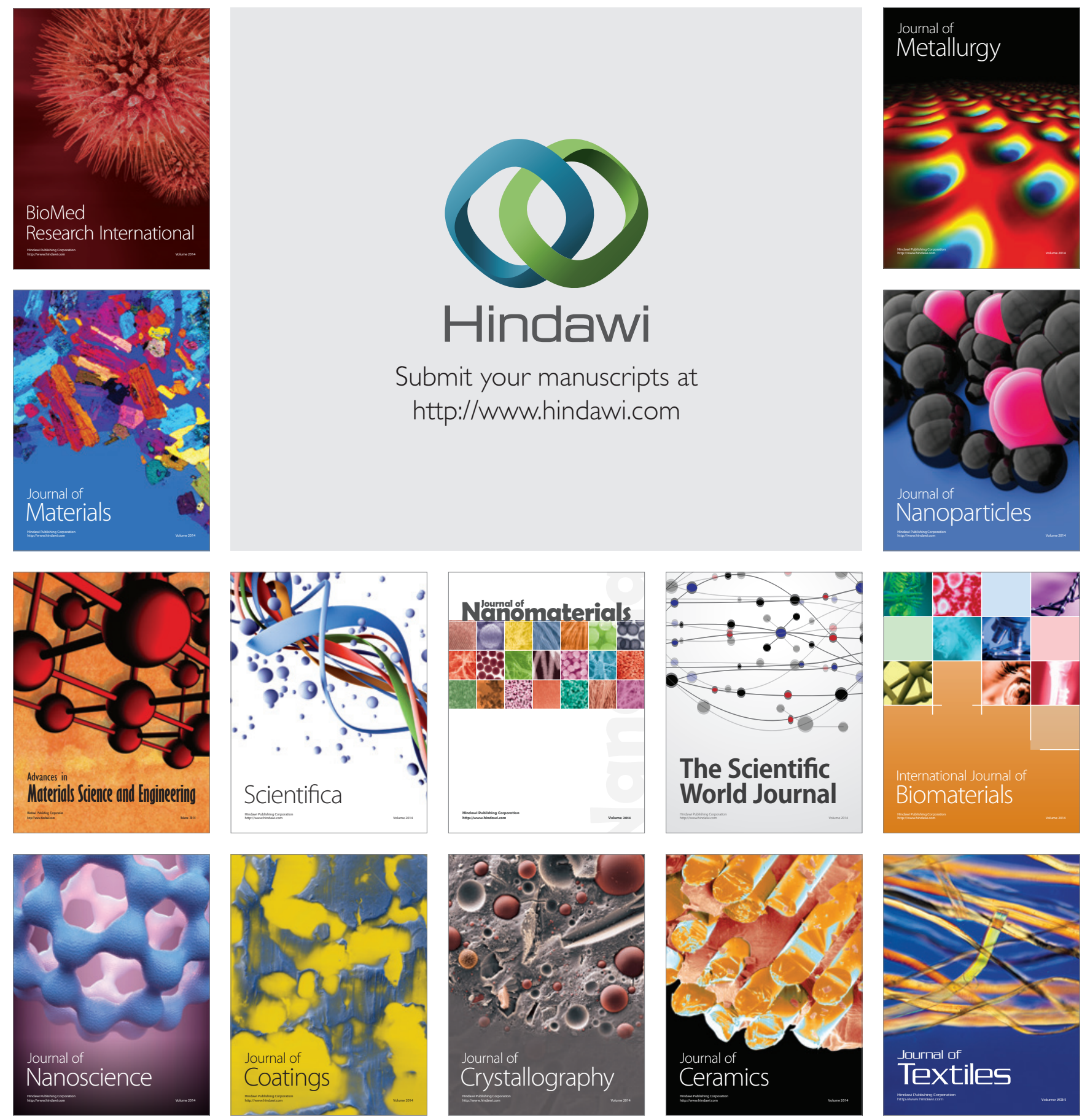